\title{
Indicator-Based Evolutionary Level Set Approximation: Mixed Mutation Strategy and Extended Analysis
}

Lai-Yee Liu ${ }^{1}$, Vitor Basto-Fernandes ${ }^{2,3(\bowtie)}$, Iryna Yevseyeva ${ }^{4}$, Joost Kok $^{1}$, and Michael Emmerich ${ }^{1}$

1 Leiden Institute of Advanced Computer Science, Leiden University,

Niels Bohrweg 1, 2333 CA Leiden, The Netherlands

emmerich@liacs.nl

2 Instituto Universitário de Lisboa (ISCTE-IUL), University Institute of Lisbon,

ISTAR-IUL, Av. das Forças Armadas, 1649-026 Lisboa, Portugal

vmbfs@iscte.pt

3 School of Technology and Management, Computer Science and Communications

Research Centre, Polytechnic Institute of Leiria, 2411-901 Leiria, Portugal

4 Faculty of Technology, De Montfort University, Gateway House 5.33, The Gateway, Leicester LE1 9BH, UK

\begin{abstract}
The aim of evolutionary level set approximation is to find a finite representation of a level set of a given black box function. The problem of level set approximation plays a vital role in solving problems, for instance in fault detection in water distribution systems, engineering design, parameter identification in gene regulatory networks, and in drug discovery. The goal is to create algorithms that quickly converge to feasible solutions and then achieve a good coverage of the level set. The population based search scheme of evolutionary algorithms makes this type of algorithms well suited to target such problems. In this paper, the focus is on continuous black box functions and we propose a challenging benchmark for this problem domain and propose dual mutation strategies, that balance between global exploration and local refinement. Moreover, the article investigates the role of different indicators for measuring the coverage of the level set approximation. The results are promising and show that even for difficult problems in moderate dimension the proposed evolutionary level set approximation algorithm (ELSA) can serve as a versatile and robust meta-heuristic.
\end{abstract}

\section{Introduction}

The problem of black box level set approximation is to find all inputs (arguments) of a function that give rise to an observed or targeted output. In general, we demand the output to be within a range or below a threshold $\epsilon \in \mathbb{R}$ and we aim to approximate the set. Given a black box objective function $f: S \rightarrow \mathbb{R}$, with $S \subset \mathbb{R}^{d}$, we search for the set $L(f \leq \epsilon)$ which is defined as:

$$
L(f \leq \epsilon):=\{\mathbf{x} \in S \mid f(\mathbf{x}) \leq \epsilon\}
$$


In the following we assume that $\mathbf{x}$ is taken from a compact domain $S$. More specifically, we will in the following look at problems where the input variables are constrained by box constraints:

$$
S=\left[\mathbf{x}_{1}^{\min }, \mathbf{x}_{1}^{\max }\right] \times \cdots \times\left[\mathbf{x}_{d}^{\min }, \mathbf{x}_{d}^{\max }\right]
$$

Problems of level set approximation occur in various disciplines of science and engineering.

- Fault Detection and Model-Based Diagnosis: The problem could be to find all possible source locations of a contamination given a model of a water distribution system [ZR07].

- Parameter Identification: Find all parameters of a system's model that can explain an observed behavior. The behavior can, for instance, be given by gene activation time series and it is used to find unknown reaction rates (propensities) in a gene regulatory network model [NE15].

- Design Engineering: Find all possible designs that comply with a prescribed behavior. For instance, different designs of building shapes that are compliant with maximum stress and with energy efficiency demands could be searched for [PCWB00, ZR07].

- De Novo Drug Discovery: Represent the space of molecular compounds that have chemical properties within a prescribed range. See for instance [vdB13]. Moreover, different low energy configurations and positions of molecules could be searched for in molecular docking problems.

This paper contributes to the development of a robust evolutionary algorithm for black box level set approximation. The steady-state algorithm ELSA ( Evolutionary Level $\underline{\text { Set }}$ Approximation) [EDK13] is tested on a broader range of problems including for the first time problems in more than two dimensions. To test the ELSA approach, we construct a set of nonlinear test problems that cover a wide range of properties and we study the geometry of the solution sets. We also study a dual mutation operator that can help to better identify disconnected components of level sets.

The paper is structured as follows: After discussing related work in Sect. 2, we describe the ELSA algorithm in Sect. 3. After this a set of benchmark problems is introduced in Sect. 4 and we summarize experimental studies on the robustness and precision of selected algorithm variants in Sect. 5. Finally, Sect. 6 concludes the paper with a summary of main results and outlook to future studies in this research line.

\section{Related Work}

Level set approximation has received some attention in numerical analysis [Set99], where it is usually used for solving explicitly given problems and not for black box formulation, but so-far little attention has been paid to targeting black box problems and population-based search heuristics for solving them. 
In practice, the use of complex simulation codes for function evaluations has increased the need for such black box enabled techniques.

As opposed to the often discussed problem of black box optimization, in level set approximation we are not in the first place interested in optimal solutions, but rather in solutions that satisfy certain criteria. The underlying assumption can be that the system's measurements are not exact and a minimization of, for instance, the deviation from the desired target could exclude possible explanations or solutions. A closely related question, related to level set approximation, is to find all solutions that are within a certain tolerance range close to the globally optimal solution [ZR07]. Moreover, approximating Pareto fronts in multiobjective optimization has much in common with level set approximation, as in both cases a set that satisfies certain conditions should be covered. However, in multi-objective optimization, the Pareto dominance relation is considered for qualification of whether a point belongs to the set to be covered relative to the position of other points in the objective space. Still, many principles of multiobjective algorithm design such as the use of indicators, population-based methods, and exploration/exploitation handling, are also of interest in the design of evolutionary level set approximation [EBN05].

A closely related work is diversity optimization, a term used by Ulrich and Thiele [UT11]. The idea of their algorithm NOAH is to find diverse sets of optimal or near optimal solutions. The algorithm NOAH lowers the threshold level gradually while evolving a population of points w.r.t. the maximization of diversity. In particular, the Solow-Polasky diversity metric [SPB93] was chosen in this context, which has several favorable theoretical properties but also requires the choice of a correlation parameter in its definition. Similar to ELSA, NOAH follows an indicator-based steady-state selection scheme, but it differs in the range of diversity indicators to be applied and in the way infeasible solutions are treated. Whereas ELSA uses augmentation, a kind of smooth penalization of infeasible solutions, in NOAH different phases of the algorithm are defined in which the constraints are gradually tightened. However, this scheme requires setting of many parameters which makes benchmarking of NOAH difficult. In our work, we use the Solow-Polasky metric, similar to NOAH. Hence ELSA can be considered as a very similar algorithm or variant of NOAH.

As opposed to diversity maximization, level set approximation seeks to find a representation of the level set. This should be expressed in the performance assessment. It has been argued in [EDK13] that if a set in $\mathbb{R}^{d}$ is approximated by a maximally diverse set, then the solution sets tend to distribute along the boundary of the level set. This would imply larger distances between solutions of the level set and gaps in the interior. In contrast, the problem of representing the level set well would rather imply closeness of the approximation set to the targeted set in the sense of minimal Hausdorff distance, meaning that all points in the targeted set should be as close as possible to (some) points in the approximation set, and vice versa, all points in the approximation set should be as close as possible to (some) points in the target set. Unfortunately, the first criterion cannot be assessed if the target set is not yet known. It is therefore, inevitable to 
use proxy indicators to assess the performance of an approximation set within the algorithm. Several such proxy indicators, including the Solow-Polasky indicator, have been discussed in [EDK13].

\section{Evolutionary Level Set Approximation (ELSA)}

ELSA is a relatively novel, simple in design, evolutionary algorithm (EA) for level set approximation. It is guided by quality indicators (QIs) that rate the fitness of a population. ELSA is a $(\mu+1)$-EA (or steady-state EA), which means that ELSA creates one child per generation and only one solution cannot survive to the next generation. Steady-state selection is commonly adopted by indicatorbased EAs (IBEAs) to circumvent computationally expensive subset selection problems [EBN05].

\subsection{Quality Indicators for Level Set Approximation}

A quality indicator (QI) assigns a single value to a level set approximation, that is a finite set $\mathrm{A} \subset S$. It should consider how many points of the level set have been found and how well they are distributed. In [EDK13] a detailed discussion is provided and here we will only summarize the most important definitions. A quality indicator is monotonous, if it grows with the number of points in the feasible set. It should also reward a good coverage of the level set. Indicators which fulfill these properties are the Augmented Average Distance $\left(\mathrm{ADI}^{+}\right)$, Augmented Solow-Polasky $\left(\mathrm{SP}^{+}\right)$, and three types of Augmented Gap indicators $\left(\mathrm{GI}^{+}\right)$: Augmented Min-Max Diversity $\left(\mathrm{GI}_{N}^{+}\right)$, Augmented Arithmetic Mean $\left(\mathrm{GI}_{\Sigma}^{+}\right)$, and Augmented Geometric Mean $\left(\mathrm{GI}_{\Pi \Pi}^{+}\right)$. In this study, only the $G I_{\Pi}^{+}$and the $\mathrm{SP}^{+}$ indicator are used, as the $\mathrm{ADI}^{+}$indicator is computationally expensive and the other augmented gap indicators had several disadvantages that were highlighted in [EDK13]. The SP indicator is defined in [SPB93] and measures the number of species in a population. This indicator has a $\theta$ parameter that scales the distance matrix and $\theta=10$ is recommended [UBT10]. Let $D(\boldsymbol{x}, Y)$ denote the (Euclidean) distance of $\boldsymbol{x}$ to the closest point in a set $Y$. The Geometric Mean is defined as $\mathrm{GI}_{\Pi}=\prod_{\boldsymbol{p} \in \mathrm{A}}^{n} D(\boldsymbol{p}, \mathrm{A} \backslash\{\boldsymbol{p}\})$, with $A$ being the approximation set that is made up of all solutions $x$ combined. In level set optimization, we only measure the diversity of the feasible subset and subtract a penalty for all infeasible points that growth proportionally with the distance to the threshold $\epsilon$. By this, each indicator can be extended to an augmented indicator which in turn can be used as a quality indicator: indicator ${ }^{+}=\operatorname{indicator}(L \cap A)-\operatorname{penalty}(A \backslash L)$. For the Gap indicator we chose penalty $(X)=\sum_{\boldsymbol{x} \in X}(\operatorname{Diameter}(S)+(f(\boldsymbol{x})-\epsilon))$, where Diameter denotes the longest distance in $S$. For the SP indicator, the penalty reads penalty $(X)=\sum_{\boldsymbol{x} \in X}(f(\boldsymbol{x})-\epsilon)$. By choosing these penalties, it is made sure that replacing an infeasible point in A by a feasible point always yields an improvement. 


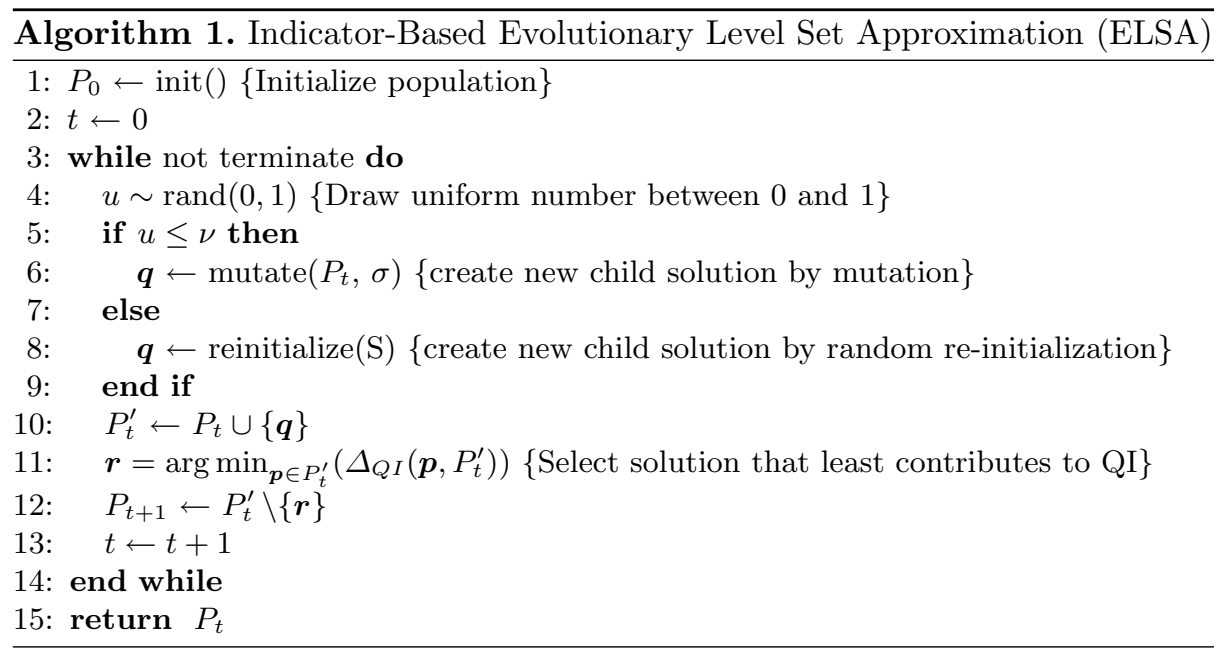

\subsection{Basic Algorithm}

Algorithm 1 describes the steps of ELSA. $P_{t}$ is the population of approximation set solutions in generation $t$. It contains the points that represent the level set. The first step in the main loop is to create the child $q \in S$, for instance by adding a small perturbation to a solution in $P_{t}$.

ELSA adopts a mixed mutation strategy, for a constant mutation probability, the algorithm either creates a child by randomly creating a new point in $S$ (random reinitialization), or by adding a perturbation to a point in $P_{t}$ (parentbased mutation). The perturbation is drawn from an i.i.d. multivariate normal distribution with a standard deviation of $\sigma$ (step-size) and mean value of zero. The default pseudo-random number generator from MATLAB 11 was used in this work, both for the normal and for the uniform distribution. The decision of which mutation to use, is based on a random number itself. The parameter $\nu \in\{0,1\}$ is the probability that a parent-based mutation is used, and $1-\nu$ is the probability of random reinitialization. The reinitialization step is a simple means to prevent the algorithm getting trapped in a local optimum or to miss a component of the level set and the setting of $\nu$ and $\sigma$ will be subject to further study in this paper. In the next step, $P_{t}^{\prime}$ denotes the temporary new population which includes solution $\boldsymbol{r}$. To keep the population size constant, in step 11 and step 12, the least contributing individual in $P_{t}^{\prime}$ is identified and discarded from $P_{t}^{\prime}$ to form the new parent population $P_{t+1}$. The contribution of a point is decided by its Quality Indicator Contribution (QIC) which, for a given quality indicator (to be maximized) is defined as: $\Delta_{\mathrm{QI}(\boldsymbol{p}, \mathrm{A})}:=\mathrm{QI}(\mathrm{A})-\mathrm{QI}(\mathrm{A} \backslash\{\boldsymbol{p}\})$. The algorithm is terminated when the number of evaluations exceeds a user-defined evaluation budget. 


\section{Benchmark Problems for ELSA}

ELSA has only been tested on benchmark problems in two dimensions in previous research. Next we propose a set of benchmark problems for more than 2 dimensions. The benchmarks are divided into two categories: simple and complex shapes. Simple shapes refer to basic geometrical objects, such as generalizations of spheres. Simple shape benchmarks are Lamé, Ellipsoid, Hollow Sphere, and Double Sphere:

$$
\begin{gathered}
L_{\text {Lamé }}(\boldsymbol{x})=\left\{\boldsymbol{x} \in[-3,3]^{d} \mid \sum_{i=1}^{d} \sqrt{\left|\frac{\boldsymbol{x}_{\boldsymbol{i}}}{3}\right|}-1 \leq 0\right\} \\
L_{\text {Ellipsoid }}(\boldsymbol{x})=\left\{\boldsymbol{x} \in[-3,3]^{d} \mid \sum_{i=1}^{d}\left(\frac{\boldsymbol{x}_{\boldsymbol{i}}}{\boldsymbol{c}_{\boldsymbol{i}}}\right)^{2}-1 \leq 0\right\}
\end{gathered}
$$

where $c=\left[\begin{array}{lll}1 & 2 & 2.5\end{array}\right]$ for 3D, and $c=\left[\begin{array}{llllllllll}1 & 2 & 2.5 & 1 & 2 & 2.5 & 1 & 2 & 2.5 & 1\end{array}\right]$ for $10 \mathrm{D}$

$$
\begin{aligned}
& L_{\text {Hollow }}(\boldsymbol{x})=\left\{\boldsymbol{x} \in[-3,3]^{d}|| \sqrt{\sum_{i=1}^{d} x_{i}^{2}-1.5} \mid \leq 0\right\} \\
& L_{\text {Double }}(\boldsymbol{x})=\left\{\boldsymbol{x} \in[-3,3]^{d} \mid\left(\sqrt{\sum_{i=1}^{d}\left(x_{i}+1\right)^{2}}-1\right) \cdot\left(\sqrt{\sum_{i=1}^{d}\left(x_{i}-1\right)^{2}}-1\right) \leq 0\right\}
\end{aligned}
$$

Complex shapes refer to engineering relevant shapes described by functions more complex than those found in the simple shapes benchmark category. The shape functions used for the complex shape benchmarks are taken from mathematical functions in multimodal optimization problems. They are used to show the performance of the Indicator-Based Evolutionary Algorithms on more realistic landscapes in terms of practical test problems. The complex shape benchmarks are Branke's Multipeak [Bra98,Kru12], Rastrigin, Schaffer [Kru12], and Vincent [vdGSB08]:

$$
\begin{array}{r}
f_{\text {Branke's }^{\prime}(\boldsymbol{x})=\frac{1}{d} \sum_{i=1}^{d}\left(1.3-g\left(x_{i}\right)\right)} \\
g\left(x_{i}\right)= \begin{cases}-\left(x_{i}+1\right)^{2}+1 & \text { if }-2 \leq x_{i}<0 \\
1.3 \cdot 2^{-8\left|x_{i}-1\right|} & \text { if } 0 \leq x_{i} \leq 2 \\
0 & \text { otherwise }\end{cases} \\
L_{\text {Branke's }}(\boldsymbol{x})=\left\{\boldsymbol{x} \in[-2,2]^{d} \mid f_{\text {Branke's }}(\boldsymbol{x}) \leq 0.4\right\}
\end{array}
$$

where this benchmark is not included as a level set benchmark for 10D

$$
L_{\text {Rastrigin }}(\boldsymbol{x})=\left\{\boldsymbol{x} \in[-4.5,4.5]^{d} \mid 10 d+\sum_{i=1}^{d}\left(x_{i}^{2}-10 \cdot \cos \left(2 \pi x_{i}\right)\right) \leq 29\right\}
$$




$$
\begin{gathered}
L_{\text {Schaffer }}(\boldsymbol{x}) \\
=\left\{\boldsymbol{x} \in[-2.5,2.5]^{d} \mid \sum_{i=1}^{d-1}\left(x_{i}^{2}+x_{i+1}^{2}\right)^{0.25} \cdot\left(\sin ^{2}\left(50 \cdot\left(x_{i}^{2}+x_{i+1}^{2}\right)^{0.1}\right)+1\right) \leq 2\right\} \\
L_{\text {Vincent }}(\boldsymbol{x})=\left\{\boldsymbol{x} \in[0.5,5]^{d} \mid-\frac{1}{d} \sum_{i=1}^{d} \sin \left(10 \cdot \ln \left(x_{i}\right)\right) \leq-0.8\right\}
\end{gathered}
$$

The difficulty of the level set benchmark depends on the level set shapes and their sizes. In general small objects are more difficult to locate, which leads to an increase in difficulty for the benchmark. The same applies for thin parts or acute angles in an object, which add a challenge when locally exploring a feasible component. If a level set has disjoint parts, it is expected that the algorithm should settle at least one solution on each disjoint part, unless the approximation set size is smaller than the amount of disjoint parts. Many of the chosen complex shape level sets have an exponential growth in the number of their disconnected component when increasing the dimensionality of the level set benchmark. Having large distances between the disjoint parts can serve as a way to test the global search capabilities of the algorithm. To this end, the difficulty of the level set benchmarks has been tailored according to the philosophy of the ELSA algorithm to not consider points in the exterior of the approximation set. Therefore, we avoid components of measure zero. The shape of the level sets can be seen in Fig. 1.

\section{Experimental Analysis}

The experiments with ELSA consist of two main parts: First part shows that implementing both global and local search is essential for black box level set benchmarks. The importance of mixed mutation strategy is measured by comparing different $\nu$ values for ELSA on 3D Ellipsoid and Vincent level set benchmark for two different $\sigma$ step-sizes (results presented in Table 1 ); In the second part, the importance of choosing the right $\sigma$ step-size is highlighted, this is seen from the experiments of ELSA on all benchmarks. These experiments show the robustness of ELSA with different $\sigma$ step-sizes on 3D and 10D benchmarks (Tables 2 and 3, respectively), where $\nu$ is set to 0.5 (a recommended parameter value derived from the mixed mutation strategy experiments). Finally, Table 4 presents a comparison on the amount of evaluations to yield a population that solely consists of feasible solutions, with respect to different step-sizes, for 3D and 10D benchmarks. The Monte Carlo Search (MCS) is included as a reference algorithm configuration in all the experiments. ELSA can easily be transformed into the MCS approach by setting $\nu$ to 0 ( $\sigma$ is irrelevant as the algorithm never produce children through parent perturbation).

The evaluation budget for a single ELSA run is set to $10 \mathrm{~K}$ for all $3 \mathrm{D}$ level set benchmarks and $100 \mathrm{~K}$ for $10 \mathrm{D}$ benchmarks. Population size $\mu=100$ is used 
for all experiments. Each configuration has been run 40 times. Several $\sigma$ stepsizes are to be defined to use in ELSA. The generic form for the $\sigma$ step-size is described below, where $d$ is the dimension:

$$
\sigma=\frac{\omega \cdot \operatorname{mean}\left(\mathbf{x}^{\max }-\mathbf{x}^{\min }\right)}{\sqrt{d}}
$$

$\sqrt{d}$ is derived from the longest diagonal of an $n$-dimensional hypercube. The chosen $\omega$ values for the $\sigma$ step-sizes are 1, 0.1, 0.01, and 0.001 (different magnitudes of 10). 3D Ellipsoid and 3D Vincent are used as the level set benchmarks to compare the results for mixed mutation strategy. They represent the core opposites of having a non-disjoint level set with Ellipsoid versus the multisegmented Vincent level set. The chosen $\nu$ values are $0,0.25,0.5,0.75$, and 1 .

The following objectives are used for the comparison of the results:

- Diversity: The Quality Indicator value of the final population.

- Eval Feasible: The amount of evaluations it takes to yield a population that solely consists of feasible solutions.

- Coverage of the final population on the level set determined by human observation.

Table 1 allows us to reason about the effect $\nu$ has in scenarios of small and big step-sizes, for single level set (Ellipsoid) and multiple level set (Vincent) problems. For the Ellipsoid problem with $\omega=0.1$, we see that the higher the $\nu$ value the better the diversity yielded with $\mathrm{GI}_{\Pi}^{+}$and $\mathrm{SP}^{+}$. This behavior is mainly explained by the fact that Ellipsoid is a single level set problem. In Vincent problem with $\omega=0.1$ both $\mathrm{GI}_{\Pi}^{+}$and $\mathrm{SP}^{+}$indicators show that intermedium values of $\nu$ (0.25 and 0.5) perform better than extreme ones such as 0 and 1 (best from worst for $\mathrm{GI}_{\Pi}^{+}$and $\mathrm{SP}^{+}: 0.25,0.5,0,0.75,1$, with $\nu=1$ being significantly worse).

For $\omega=0.01$, both $\mathrm{GI}_{\Pi}^{+}$and $\mathrm{SP}^{+}$indicators in the Ellipsoid problem also reveal that intermedium values of $\nu$ such as 0.5 and 0.75 perform better than extreme ones $\left(\mathrm{GI}_{\Pi}^{+}\right.$best to worst: $0.5,0.75,0.25,0,1$; and SP: $0.75,0.5,0.25$, $0,1)$. A similar conclusion can be made for the Vincent problem with $\omega=0.01$, intermedium values of 0.25 and 0.5 of $\nu$ allow for best $\mathrm{GI}_{\Pi}^{+}$and $\mathrm{SP}^{+}$results (best to worst, $\mathrm{GI}_{\Pi}^{+}$and $\left.\mathrm{SP}^{+}: 0.25,0.5,0,0.75,1\right)$.

Results from Table 1 show that the tuning/trade-off settings allowed by ELSA mixed mutation strategy, is essential for the exploration and exploitation of single and multiple level set problems. For the test on different values of $\nu$ it can be observed that higher values of $\nu$ have priority, in case of simple problems with connected level sets (Ellipsoid). On the contrary, a value of $\nu$ that lies between 0 and 1 should be chosen, if the level set is disconnected (Vincent). There is an optimal setting of $\nu$ which is in the middle between the two extremes. This makes sense, as in the multimodal case it is important to explore (find new components of the level set by reinitialization), but also one has to distribute points well in the found level set components (parent based mutation). 
Table 1. The diversity of the final populations from ELSA with $\omega=0.1$ and $\omega=0.01$ for $\sigma$ step-size and different $\nu$ values on selected 3D level set benchmarks.

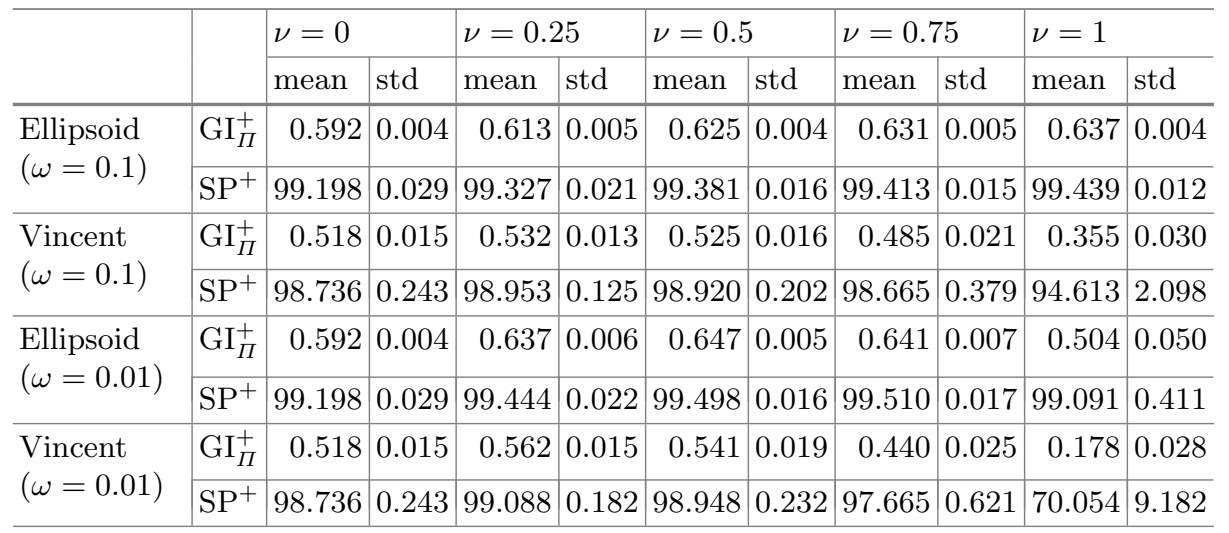

Experiment results in Table 2 show that ELSA is able to consistently find entirely feasible populations with high diversity on all 3D level set benchmarks for almost all the tested $\sigma$ step-sizes. Even MCS can produce relatively good results with the exception of Lamé benchmark which proves to be too difficult to find feasible solutions with purely random search. The results from $\omega=1$ resemble the results of MCS, thus it can be considered a too large step-size. ELSA with $\omega=0.1$ and $\omega=0.01$ are most successful at finding diverse approximation sets, where the results with $\omega=0.1$ have noticeably better diversity in 3D Schaffer. Results from $w=0.001$ however show a decline in diversity which indicates that this $\sigma$ step-size is too small for the level set benchmarks. Similar patterns between the different step-sizes are reflected in the 10D level set benchmarks (See Table 3). Again, the configurations with $\omega=0.1$ and $\omega=0.01$ are observed to be most suited in general for these type of black-box level set benchmarks. However, the limitations of ELSA are revealed in higher dimensions as it struggles with finding an entirely feasible set for Lamé, Rastrigin and Schaffer regardless of $\sigma$ step-size. MCS and ELSA with $\omega=1$ are the most severe cases whereby they cannot even find any entirely feasible populations. For the other $10 \mathrm{D}$ benchmarks, ELSA with $\omega=0.1$ or $\omega=0.01$ find diverse populations where the results from Solow-Polasky on Ellipsoid and Hollow Sphere are near optimal in measurement.

Table 4 experiments compare 3D and 10D convergence to the level set, it shows that the setting of the step size parameter is crucial not only for finding sets with a good coverage, but also for finding the components of the level set. Step size settings are more critical in the high dimensional case and in the future work automatic adaptation of the step size should be developed. 
Table 2. The diversity of the final populations from the tested algorithm configurations on the 3D level set benchmarks.

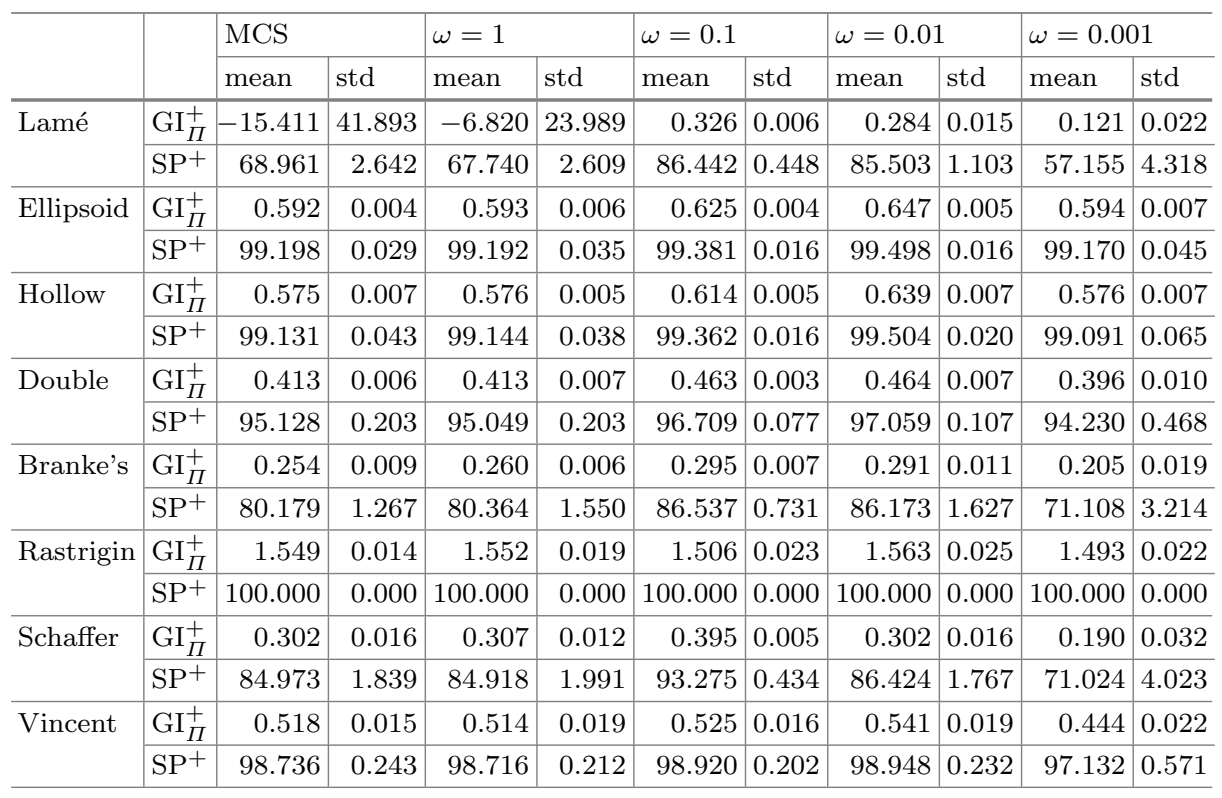

Table 3. The diversity of the final populations from the tested algorithm configurations on the 10D level set benchmarks.

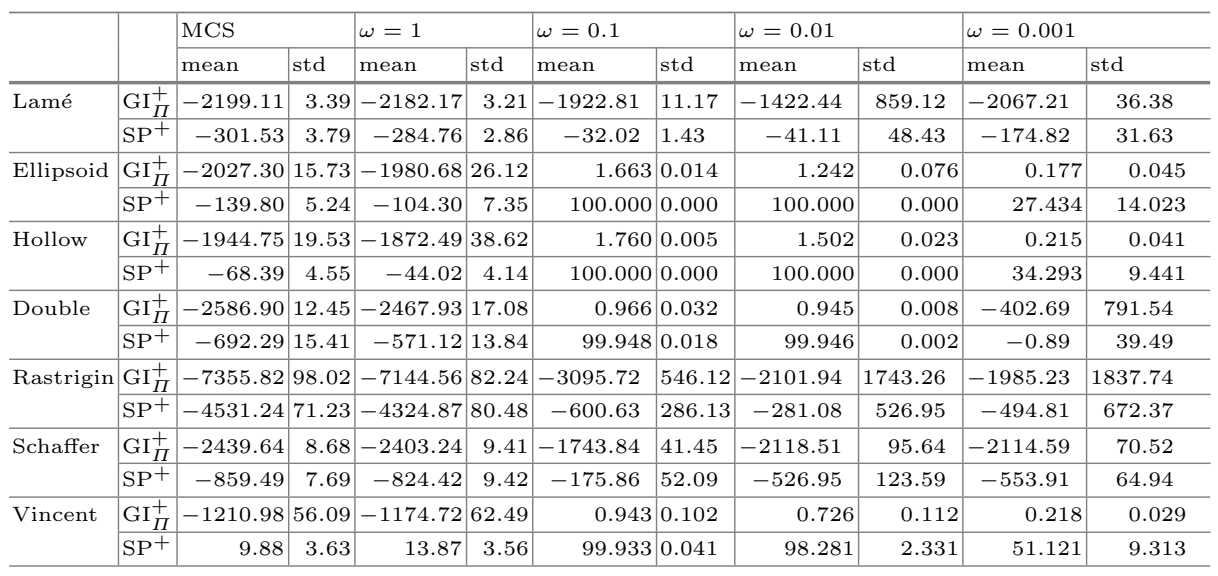

Figure 1 represents the final populations of ELSA, generated with $\mathrm{GI}_{\Pi}^{+}$under the setting of $\nu=0.5$ and $\omega=0.1$, for 3D benchmarks of Lamé (a), Ellipsoid (b), Branke's Multipeak (c), Rastrigin (d), Schaffer (e) and Vincent (f). The populations are selected in a way that their measured diversity comes closest to the average diversity found over all runs. The populations from $\mathrm{SP}^{+}$have a 
Table 4. Eval Feasible of the results from the tested algorithm configurations. Eval $_{\text {Feasible }}$ is calculated as the average of the results from $\mathrm{GI}_{\Pi}^{+}$and $\mathrm{SP}^{+}$combined. The "-" symbol marks a configuration that contains at least one population that does not solely consist of feasible solutions.

\begin{tabular}{|c|c|c|c|c|c|c|c|c|c|c|c|c|c|c|c|c|}
\hline & \multicolumn{10}{|l|}{$3 \mathrm{D}$} & \multicolumn{6}{|l|}{$10 \mathrm{D}$} \\
\hline & \multicolumn{2}{|l|}{ MCS } & \multicolumn{2}{|l|}{$\omega=1$} & \multicolumn{2}{|c|}{$\omega=0.1$} & \multicolumn{2}{|c|}{$\omega=0.01$} & \multicolumn{2}{|c|}{$\omega=0.001$} & \multicolumn{2}{|c|}{$\omega=0.1$} & \multicolumn{2}{|c|}{$\omega=0.01$} & \multicolumn{2}{|c|}{$\omega=0.001$} \\
\hline & mean & std & mean & std & mean & std & mean & std & mean & std & mean & std & mean & std & mean & std \\
\hline Lamé & - & - & - & - & 1067 & 113 & 3911 & 137 & 957 & 238 & - & - & - & - & - & - \\
\hline Ellipsoid & 1030 & 101 & 1044 & 93 & 468 & 40 & 454 & 49 & 456 & 49 & 3045 & 493 & 9022 & 2436 & 40474 & 17868 \\
\hline Hollow & 1279 & 122 & 21252 & 119 & 539 & 46 & 5492 & 46 & 488 & 60 & 2715 & 362 & 7852 & 2245 & 29867 & 12113 \\
\hline Double & 2570 & 278 & 2542 & 255 & 686 & 68 & 8622 & 75 & 642 & 100 & 4377 & 473 & 13679 & 3507 & - & - \\
\hline Branke's & 4488 & 468 & 4105 & 419 & 969 & 89 & 9764 & 109 & 758 & 127 & & & & & & \\
\hline Rastrigin & 1043 & 106 & \begin{tabular}{|l|l|} 
& 1062 \\
\end{tabular} & 93 & 710 & 66 & 6465 & & 453 & 42 & & - & - & - & - & - \\
\hline Schaffer & 6316 & 663 & 6247 & 586 & 1101 & 120 & 862 & 135 & 833 & 143 & & - & - & - & D & 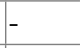 \\
\hline Vincent & 2852 & 309 & 2843 & 283 & 1086 & 91 & 1659 & & 656 & 77 & 3152 & 534 & 2694 & 674 & 5946 & 2991 \\
\hline
\end{tabular}

tendency for solutions to reside on the boundary of the level set which is not always a desired behavior when taking practical applications into account. While figures labeled with 1 (for example a1) represent 3D plots of the level sets, figures labeled with 2 represent a $2 \mathrm{D}$ projection of the $3 \mathrm{D}$ plots (for example a2). The level sets are depicted in light gray and are semi-transparent. In the 3D view, the RGB-value (converted into gray scale) of a solution maps to the $\left(x_{1}, x_{2}, x_{3}\right)$ coordinate of the solution with respect to the search space. In the orthographic view, gray scale coding is used to determine the location of the solution with respect to the $x_{3}$-axis.

By visual inspection of Fig. 1 we can state that the acute parts of Lamé are too challenging for ELSA to evenly distribute solutions over them. The populations are well-spread on simple shape benchmarks like Ellipsoid (while not depicted, it also holds for Hollow Sphere and Double Sphere). Branke's Multipeak has each level set component covered by solutions for both quality indicators, but the result from $\mathrm{GI}_{\Pi}^{+}$does not balance the number of points evenly across the components. For Rastrigin the majority of the solutions are settled on the center raster-like structure, however ELSA is able to settle some solutions on the outer rim disjoint parts. 3D Schaffer has similarities to Lamé in terms of general shape and the same problems with ELSA are encountered, but ELSA manages to distribute solutions on the exterior parts to some degree despite the lack of connectivity. 3D Vincent has 64 disjoint level set parts and neither population can cover them all, although the overall diversity is good. 


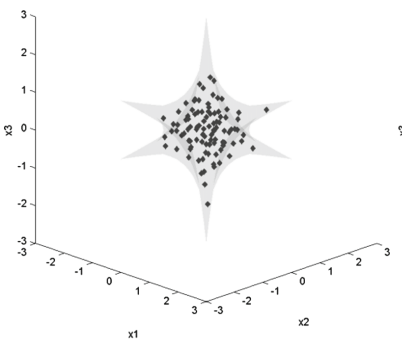

(a1) Lamé: 0.326

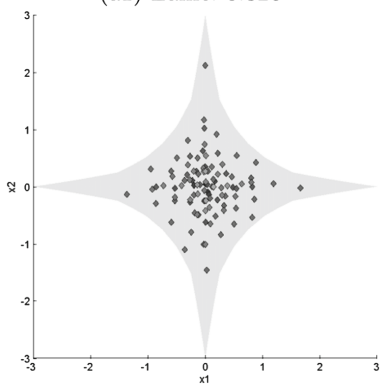

(a2)

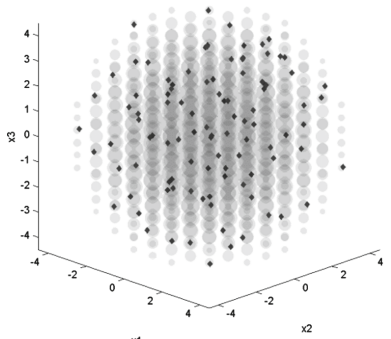

(d1) Rastrigin: 1.506

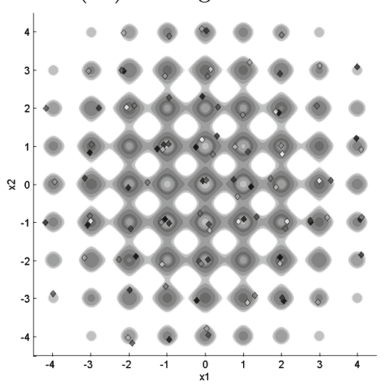

(d2)

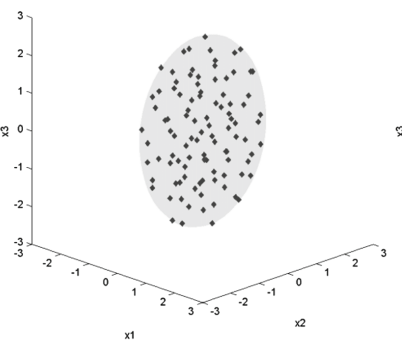

(b1) Ellipsoid: 0.625

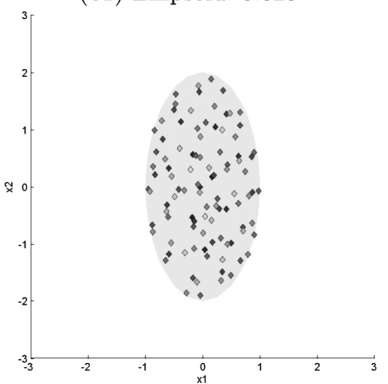

(b2)

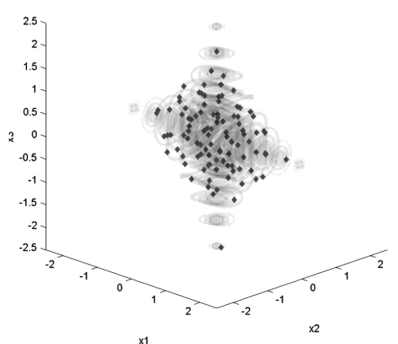

(e1) Schaffer: 0.395

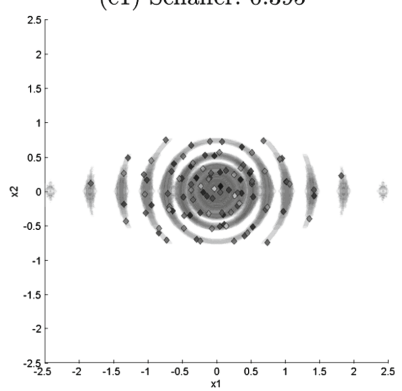

(e2)

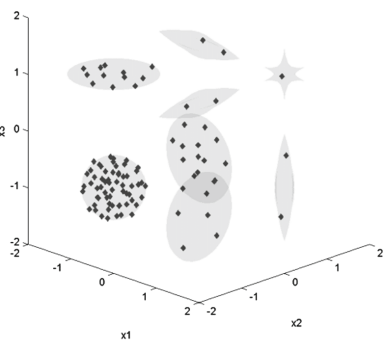

(c1) Branke's: 0.295

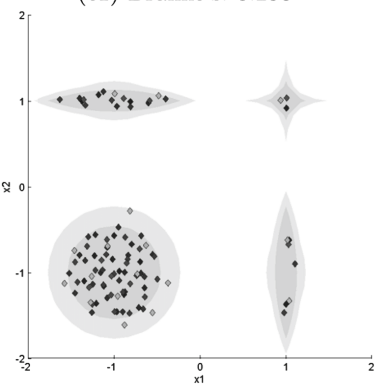

(c2)

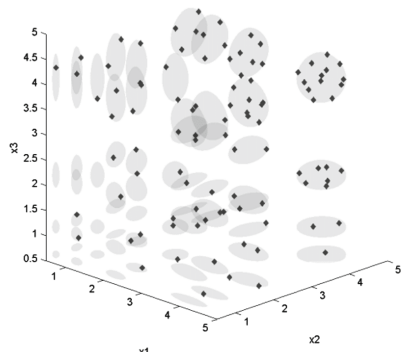

(f1) Vincent: 0.525

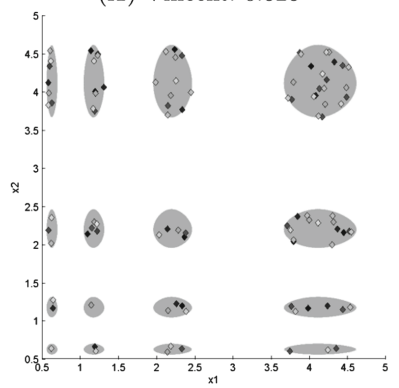

(f2)

Fig. 1. Visualization of results on $3 \mathrm{D}$ benchmark achieved with $\mathrm{GI}_{\Pi}^{+}$indicator. 


\section{Summary and Outlook}

The current study shows that a mixed strategy of random reinitialization and parent-based mutation is preferable to using only one of these strategies, because it allows to explore and to refine at the same time, thereby minimizing the risk that a component of the level set is overlooked. This mixed strategy might be considered preferable, as we lose performance for the sake of reliability.

In contrast to previous work where only $2 \mathrm{D}$ problems were addressed, in our study ELSA performance is assessed for low (3D) and high dimensional level sets (10D). This study allowed to conclude that step size parameter is crucial (specially in higher dimensions) to get both good coverage and to find the components of the level set. Automatic adaptation of the step size (Selfadaptive $\sigma$ ) should be developed to adapt the algorithm parameters to problem specific properties.

Although ELSA has revealed very good performance in simple shape benchmarks such as Ellipsoid for low and high dimensions, there is still space for improvement in shapes with acute parts such as Lamé, Rastrigin and Schaffer, specially in high dimensions. To this end, new selection and cross-over operations can be designed for ELSA.

There are still some ways that can be tried to improve the performance of ELSA: The current version of ELSA does not utilize cross-over, that might help to improve the level set coverage (close gaps). However, it might also be disruptive, when applied to individuals from different components of the level set. Moreover, creating more than one children or introduction of mating selection could be beneficial for improving the quality of results, but would also significantly increase computational costs of an iteration.

In contrast to evolutionary algorithms AIS feature a variable population size and they also have some inherent mechanisms for diversity maintenance, which makes them a promising technique for level set approximation [CZ06].

\section{References}

[Bra98] Branke, J.: Creating robust solutions by means of evolutionary algorithms. In: Eiben, A.E., Bäck, T., Schoenauer, M., Schwefel, H.-P. (eds.) PPSN 1998. LNCS, vol. 1498, pp. 119-128. Springer, Heidelberg (1998). doi:10.1007/BFb0056855

[CZ06] Coelho, G.P., Von Zuben, F.J.: omni-aiNet: an immune-inspired approach for omni optimization. In: Bersini, H., Carneiro, J. (eds.) ICARIS 2006. LNCS, vol. 4163, pp. 294-308. Springer, Heidelberg (2006). doi:10.1007/ 11823940_23

[EBN05] Emmerich, M., Beume, N., Naujoks, B.: An EMO algorithm using the hypervolume measure as selection criterion. In: Coello Coello, C.A., Hernández Aguirre, A., Zitzler, E. (eds.) EMO 2005. LNCS, vol. 3410, pp. 62-76. Springer, Heidelberg (2005). doi:10.1007/978-3-540-31880-4_5 
[EDK13] Emmerich, M.T.M., Deutz, A.H., Kruisselbrink, J.W.: On quality indicators for black-box level set approximation. In: Tantar, E., Tantar, A.A., Bouvry, P., Del Moral, P., Legrand, P., Coello, C.A.C., Schütze, O. (eds.) EVOLVE-A Bridge Between Probability, pp. 157-185. Set Oriented Numerics and Evolutionary Computation. Springer, Heidelberg (2013)

[Kru12] Kruisselbrink, J.W.: Evolution strategies for robust optimization. Ph.D. thesis, Leiden Institute of Advanced Computer Science (LIACS), Faculty of Science, Leiden University (2012)

[NE15] Nezhinsky, A., Emmerich, M.T.M.: Parameter identification of stochastic gene regulation models by indicator-based evolutionary level set approximation. In: Proceedings of EVOLVE - A Bridge Between Probability, Set-Oriented Numerics, and Evolutionary Computation, Iasi, June 2015. Springer, Heidelberg (2015, in print)

[PCWB00] Parmee, I.C., Cvetković, D., Watson, A.H., Bonham, C.R.: Multiobjective satisfaction within an interactive evolutionary design environment. Evol. Comput. 8(2), 197-222 (2000)

[Set99] Sethian, J.A.: Level Set Methods and Fast Marching Methods: Evolving Interfaces in Computational Geometry, Fluid Mechanics, Computer Vision, and Materials Science, vol. 3. Cambridge University Press, Cambridge (1999)

[SPB93] Solow, A., Polasky, S., Broadus, J.: On the measurement of biological diversity. J. Environ. Econ. Manag. 24(1), 60-68 (1993)

[UBT10] Ulrich, T., Bader, J., Thiele, L.: Defining and optimizing indicator-based diversity measures in multiobjective search. In: Schaefer, R., Cotta, C., Kołodziej, J., Rudolph, G. (eds.) PPSN 2010. LNCS, vol. 6238, pp. 707717. Springer, Heidelberg (2010). doi:10.1007/978-3-642-15844-5_71

[UT11] Ulrich, T., Thiele, L.: Maximizing population diversity in single-objective optimization. In: Proceedings of the 13th Annual Conference on Genetic and Evolutionary Computation, pp. 641-648. ACM (2011)

[vdB13] van der Burgh, B.: An evolutionary algorithm for finding diverse sets of molecules with user-defined properties. Technical report (2013)

[vdGSB08] van der Goes, V., Shir, O.M., Bäck, T.: Niche radius adaptation with asymmetric sharing. In: Rudolph, G., Jansen, T., Beume, N., Lucas, S., Poloni, C. (eds.) PPSN 2008. LNCS, vol. 5199, pp. 195-204. Springer, Heidelberg (2008). doi:10.1007/978-3-540-87700-4_20

[ZR07] Zechman, E.M., Ranjithan, R.S.: Generating alternatives using evolutionary algorithms for water resources and environmental management problems. J. Water Resour. Plan. Manag. 133(2), 156-165 (2007) 